\title{
RESEARCH
}

\section{Student use of self-directed learning time in an undergraduate medical curriculum}

\author{
James Gould MD ${ }^{\dagger \dagger}$, lain Arseneau MD†, Stephen Dalziel MD ${ }^{1 \dagger}$, Harrison Petropolis MD ${ }^{1 \dagger}$, \\ Karen Mann BN MSc PhD² \\ ${ }^{1}$ Class of 2015, Faculty of Medicine, Dalhousie University \\ ${ }^{2}$ Division of Medical Education, Faculty of Medicine, Dalhousie University \\ ${ }^{\dagger}$ Authors were equal contributors
}

\begin{abstract}
Introduction: In 2010, Dalhousie University implemented a new MD curriculum, placing an emphasis on self-directed learning (SDL) time. This study sought to understand how students use this time and whether they would benefit from more structure during SDL. We hypothesized that students spend significant amounts of SDL time on nonacademic activities and would prefer to have more specific guidance and tasks.

Methods: Pre-clerkship medical students at Dalhousie ( $n=223)$ were sent an online survey consisting of 18 questions using a combination of Likert scales, and text boxes for qualitative responses. Chi-square analysis was performed for each survey question.

Results: Eighty-five percent ( $n=93)$ of medical students responded that time scheduled for SDL was sufficient ( $p<0.001)$ and $67 \%$ ( $n=73)$ responded that they would benefit from more specific guidance and tasks during SDL time ( $p<0.001)$. Forty-five percent responded that they "rarely" spent SDL time on non-academic activities ( $n=49)$, however only $14 \%$ ( $n=15)$ responded "most of the time" ( $p<0.001)$.

Conclusion: The majority of respondents used SDL time for academic activities but felt they would benefit from more specific guidance and tasks. This is inconsistent with our hypothesis that students are spending significant amounts of SDL time on non-academic activities, but supports our hypothesis that students would prefer more structure.
\end{abstract}

Tn September 2010, Dalhousie University unveiled a new curriculum for its MD program. One of the major changes in this curriculum was the implementation of self-directed learning (SDL) time. This is time during which the school may not schedule other activities, and students are encouraged to use this time to address their own specific learning needs. Gibbons describes "SDL is any increase in knowledge, skill, accomplishment, or personal development that an individual selects and brings about by his or her own efforts using any method in any circumstances at any time". ${ }^{1}$

Spencer and Jordan outlined key principles of SDL, which include diagnosing learning needs, formulating goals, identifying resources, implementing appropriate activities, and evaluating outcomes. ${ }^{2}$ These principles are in keeping with the adult learner who wants focused, relevant content that can be directly applied to practice.

Canadian medical schools strive to produce physicians who will display the core competencies outlined by CanMEDS. ${ }^{3}$ One of those competencies is that of "life-long learner". Learning can be difficult once the student has left the confines of a structured academic environment; however, medical knowledge is expanding rapidly highlighting the need for continued education. SDL has been proposed as a way to better prepare student physicians to become life-long learners and is increasingly prevalent in medical education. ${ }^{4-6}$ However, the existing literature suggests only a comparable, or at best moderate improvement in knowledge when students use SDL as compared to more traditional approaches. ${ }^{7}$ In general, SDL was facilitated by providing study guides or online-modules that allow students to work at their own pace and on their own time while still providing structure. ${ }^{8,9}$

There have been few studies reported on how students use SDL time. One survey conducted at a medical institution in Saudi Arabia found that greater than half of their students responded that they used SDL time for non-academic activities. ${ }^{10}$ Recent evidence has also suggested that learning may improve when some direction is given to "self-regulated" study time. ${ }^{11}$ In this model, students are able to control the speed or rate of their learning but are provided direction with regards to content or their learning. Building on this framework the objectives of this study were to [1] understand how first and second year students in the Dalhousie undergraduate medicine program use their protected SDL time and [2] whether students would benefit from more guidance on how to best utilize this time. We 
predicted that a) students spend significant amounts of SDL time on non-academic activities (considered in our study to be any activity that is not directly related to the medical curriculum or objectives) and b) would prefer to have more guided tasks during SDL time.

\section{Methods}

\section{Study Design}

Dalhousie University is located in Halifax, Nova Scotia, Canada and offers a 4-year Doctor of Medicine (MD) program. The study included first and second year medical students who are in their pre-clerkship years of training. Third and fourth year clerkship students were excluded as these years consist mostly of clinical rotations, and at the time of this study were largely unchanged from previous curriculum iterations. The pre-clerkship curriculum is a mix of case-based learning (CBL), didactic lecture, and SDL. During the study period the first and second year classes at Dalhousie University Medical School comprised 223 students. A convenience sample was collected from the total population $(\mathrm{n}=109)$. This study received approval from the Dalhousie University Health Sciences Research Ethics Board.

The study was conducted as a cross-sectional survey design using Dalhousie's endorsed online survey tool, Opinio. Each eligible participant was sent a secure explanatory email containing a link to access the survey using an online survey tool. The first webpage provided the rationale for the survey and required participants to click a check box to continue, indicating informed consent. Participation in the survey remained anonymous and students were able to withdraw from the study up until submission. Students were only able to complete the survey once. The survey consisted of 18 questions (Supplemental File 1, online) using a combination of 5-point Likert scaling, and text boxes for qualitative responses. Survey questions were developed by the authors. We sought to ensure that all major components of the pre-clerkship curriculum (electives, tutorials, labs, lectures) were represented. The draft survey was reviewed by a medical educator for completeness and clarity, and revisions were made based on the feedback received. Participants completed the survey in May-June of 2013. Two electronic reminders were sent at 1 week intervals between the start and end dates of the study.

\section{Data Analysis}

Data analysis was performed using GraphPad Prism 6 software. A Chi-square analysis was performed for each survey question with $P$ values less than 0.05 required for significance. Questions one, two, and three used the options strongly disagree, disagree, neutral, agree, or strongly agree. For the purposes of the statistical analysis, strongly disagree and disagree were grouped as negative responses, neutral remained neutral, and agree and strongly agree were grouped as positive responses. Significant differences between these three groups were explored. For questions four through eleven, the possible options were never, rarely, half of the time, most of the time, and all of the time. No grouping was performed for these prompts. Questions twelve through eighteen consisted of qualitative data, collected in the form of text boxes. As such, no data analysis was performed, but these comments were used to guide our discussion, and develop hypotheses for potential follow-up studies. Question 6 was erroneously included in duplicate in our survey. For the sake of transparency, the results for each of the two inclusions were analyzed as if they were separate questions.

\section{Results}

Of the 223 students invited to participate, 123 people opened the survey, and 109 completed it, giving a response rate of $48.9 \%$. The results for statements $1-3$, which relate to the students satisfaction with SDL, are summarized in Table 1. The results for statements 4-11, which explore how students use their SDL time, are summarized in Table 2.

In Table 1, we see that students felt that time allocated for SDL was sufficient $(p<0.001)$, but that they would benefit from more guidance during SDL time $(\mathrm{p}<0.001)$ Furthermore when responses to the question "I would benefit from the provision of more specific guidelines and tasks to be used during SDL" were grouped into negative (strongly disagree and disagree), neutral, and positive (strongly agree and agree), 67\% $(n=73)$ responded positively, 9\% $(n=10)$ had a neutral stance, while $24 \%(n=26)$ responded negatively $(\mathrm{p}<0.005)$.

When responses for "If time allocated for SDL were increased then I would spend this extra time on academic activities" were grouped into negative (strongly disagree and disagree), neutral, and positive (strongly agree and agree), the differences were no longer significant $(\mathrm{p}=0.4346)$.

Table 2 indicates that students spend significant amounts of SDL time on various academic activities, and that students are not spending the majority of their SDL time on non-academic activities $(\mathrm{p}<0.001)$.

Qualitative responses further explained the quantitative survey results. Comments indicating that students used SDL time on non-academic activity included: "Honestly, I usually come home, make sure my cases are done, and take a nap" and "If I haven't 


\begin{tabular}{|c|c|c|c|c|c|c|c|c|}
\hline \multirow[t]{2}{*}{ Question } & \multicolumn{8}{|c|}{ Responses - n (\%) } \\
\hline & $\begin{array}{l}\text { Strongly } \\
\text { Disagree }\end{array}$ & Disagree & Neutral & Agree & $\begin{array}{l}\text { Strongly } \\
\text { Agree }\end{array}$ & Total & $\begin{array}{c}\text { Sample } \\
\text { SD }\end{array}$ & P-value \\
\hline $\begin{array}{l}\text { Time allocated for SDL is } \\
\text { sufficient }\end{array}$ & $0(0)$ & $6(5.5)$ & $10(9.2)$ & 71 (65.1) & $22(20.2)$ & 109 & 28.66 & $<0.001$ \\
\hline $\begin{array}{l}\text { If the allocated time for SDL } \\
\text { is increased then I will spend } \\
\text { more time on academic } \\
\text { activities }\end{array}$ & $8(7.3)$ & $34(31.2)$ & $36(33.0)$ & $23(21.1)$ & $8(7.3)$ & 109 & 13.54 & $<0.001$ \\
\hline $\begin{array}{l}\text { I would benefit from the } \\
\text { provision of more specific } \\
\text { guidance and tasks to be used } \\
\text { during SDL time }\end{array}$ & $5(4.6)$ & $21(19.3)$ & $10(9.2)$ & $37(33.9)$ & $36(33.0)$ & 109 & 14.62 & $<0.001$ \\
\hline
\end{tabular}

Table 1. Dalhousie medical students response to appropriateness of self-directed learning (SDL) in pre-clerkship training.

finished the case, then I'll work on it. Otherwise I treat it as any other time off". Responses collected that indicate students complete SDL during unprotected time include: "[I] Prioritize many commitments of our schedule...[I] participate in non-academic activities that would otherwise be challenging to be involved in (after 4:30 or on the weekends); then I would do my SDL after 4:30 or on the weekends". Some responses related to variability in usage of SDL time included: "[Allocation of SDL time] depends on what day of the week it is, and the task at hand". "Early in the week I use it for case preparation. Later in the week I review lectures" and "Early in the unit, I spend more SDL time on non-academic activities (about $1 / 2$ ) and the rest I use in preparation for case tutorials. As an exam approaches I use SDL time for review and tend to use all allocated time for school work." Finally, responses that indicate students desire more guidance in their SDL include: "Lack of guidance leads to lots of wasted SDL time", "It is really nice to have time to spend to properly learn material at my own pace. I would agree however, that some guidance as to how to use the time would likely benefit many students."

\section{Discussion}

The objectives of this study were to understand how first and second year students in the Dalhousie undergraduate medicine program use their protected SDL time and whether students would benefit from more guidance on how to best utilize this time.

\section{Use of SDL time}

We hypothesized that students use a significant amount of their protected time for non-academic activities. However, the results suggest that most students do use SDL time for academic activity. A significant proportion of students reported using SDL time for
CBL preparation $(46.8 \%$ responded with 'most of the time, $38.53 \%$ responded' half of the time'). CBL tutorials are two-hour group learning sessions that occur three times weekly in the first and second year curriculum. These sessions require that students review preparation material and answer guiding questions pertaining to a patient/clinical scenario prior to the session. It seems justified that students would allocate significant SDL time to CBL preparation as it is generally considered "mandatory" by students to do some level of preparation for these sessions in order to facilitate group discussion.

\section{SDL during unprotected time}

Despite the reported use of SDL time for CBL preparation, many comments collected in the survey suggest that students are using protected SDL time for non-academic activity with the intent of doing SDL during an "un-protected time" such as the evenings or weekend.

These qualitative results indicate that many students feel that SDL can be completed at any time they choose. However protected SDL time has been implemented in place of traditional didactic lecture time. In the traditional model there would still be an expectation that students do some form of "SDL" or "study" outside of lecture. Therefore, while the intent may be that students will study more if given this additional protected SDL time, this was not previously shown to be the case. ${ }^{10}$ Rather, the provision of this extra SDL time may simply provide more flexibility in scheduling study time as well as non-academic activities, possibly at the expense of didactic teaching or other structured curriculum activities.

\section{Variability in SDL use}

Based on qualitative responses it appears that student allocation of SDL time may be variable, depending on the day of the week, as well as the proximity to unit exams. One possible solution would be that SDL time 


\begin{tabular}{|c|c|c|c|c|c|c|c|c|}
\hline \multirow[b]{2}{*}{ Question } & \multicolumn{8}{|c|}{ Responses - n (\%) } \\
\hline & Never & Rarely & $\begin{array}{l}\text { Half the } \\
\text { time }\end{array}$ & $\begin{array}{l}\text { Most of } \\
\text { the time }\end{array}$ & $\begin{array}{l}\text { All of the } \\
\text { time }\end{array}$ & Total & Sample SD & P-value \\
\hline $\begin{array}{l}\text { I spend SDL allocated time for case- } \\
\text { based learning preparation }\end{array}$ & $2(1.8)$ & 11 (10.1) & $42(38.5)$ & $51(46.8)$ & $3(2.8)$ & 109 & 23.04 & $<0.001$ \\
\hline $\begin{array}{l}\text { I spend SDL allocated time for lecture } \\
\text { objectives }\end{array}$ & $6(5.6)$ & $52(48.1)$ & $33(30.6)$ & 17 (15.7) & $0(0.0)$ & 108 & 21.13 & $<0.001$ \\
\hline $\begin{array}{l}\text { I spend SDL allocated time for self- } \\
\text { study individually }\end{array}$ & $2(1.8)$ & 37 (33.9) & $35(32.1)$ & $33(30.3)$ & $2(1.8)$ & 109 & 18.13 & $<0.001$ \\
\hline $\begin{array}{l}\text { I spend SDL allocated time preparing } \\
\text { for lab }\end{array}$ & $5(4.6)$ & $67(61.5)$ & $26(23.9)$ & 11 (10.1) & $0(0.0)$ & 109 & 27.09 & $<0.001$ \\
\hline $\begin{array}{l}\text { I spend SDL allocated time preparing } \\
\text { for Professional Competencies }\end{array}$ & 17 (15.6) & $56(51.4)$ & $29(26.6)$ & $6(5.5)$ & $1(0.9)$ & 109 & 21.95 & $<0.001$ \\
\hline $\begin{array}{l}\text { I spend SDL allocated time preparing } \\
\text { for or attending electives }\end{array}$ & 11 (10.1) & $36(33.0)$ & $43(39.5)$ & 17 (15.6) & $2(1.8)$ & 109 & 17.20 & $<0.001$ \\
\hline $\begin{array}{l}\text { I spend SDL allocated time for non- } \\
\text { academic activities }\end{array}$ & $3(2.8)$ & $49(45.0)$ & $42(38.5)$ & 15 (13.8) & $0(0.0)$ & 109 & 22.49 & $<0.001$ \\
\hline $\begin{array}{l}\text { I spend SDL time for self-study } \\
\text { individually* }\end{array}$ & $2(1.9)$ & $26(24.1)$ & 39 (36.1) & 39 (36.1) & $2(1.9)$ & 108 & 18.66 & $<0.001$ \\
\hline I spend SDL time for group discussions & $48(44.4)$ & $50(46.3)$ & $9(8.3)$ & $1(0.9)$ & $0(0.0)$ & 108 & 25.26 & $<0.001$ \\
\hline
\end{tabular}

Table 2. Dalhousie medical students use of their self-directed learning (SDL) time in pre-clerkship training. *This question was duplicated in the survey and both responses were analyzed and presented for transparency.

could be slowly increased in quantity throughout the unit, with didactic teaching front loaded in the unit and SDL time for studying closer to end of the unit during exams. However, further study of variability in students' SDL use over the semester and in proximity to unit exam would be needed before any curriculum change would be recommended.

\section{Guiding SDL time}

The second objective of this study was to determine if students perceived they would benefit from guidance on how to utilize SDL time. The results of this study, both qualitative and quantitative clearly demonstrate that students would prefer some form of guidance in order to direct the use of their SDL time. Curriculum structured in a way that included guidance or objectives for students to utilize during their SDL would be better defined as "directed, self regulated learning". It appears that medical students at Dalhousie University would prefer this additional direction. Currently in the curriculum there are a set of objectives linked to each lecture, CBL case and lab as well as overall objectives for the unit. If these objectives could be improved upon and be more specific, then students may feel like they have more direction in their SDL time. However, further research into these suggestions and this type of curriculum would be needed to determine its effectiveness. It would be important for those responsible for curriculum decisions to take this into consideration when making curriculum changes.

Medical students are responsible for learning a vast amount of material at a rapid pace. There have been attempts to identify students who are most likely to succeed in an SDL environment by measuring SDL readiness using the scale developed by Guglielmino; however there have been questions about its applicability for medical students. ${ }^{6,12-13}$ In addition, it has been demonstrated that SDL readiness can actually regress during medical education. ${ }^{14}$ Therefore, it may not be a matter of determining whether or not SDL is effective, or which students are more prepared for SDL, but instead of providing adequate support and guidance for students during SDL time, while still cultivating life-long learning skills. Indeed, there is literature to support that students may need more support in terms of managing their SDL time. ${ }^{15}$

Previous studies at Dalhousie have indicated that a hybrid problem-based learning (PBL) curriculum contributed to students' SDL skills. ${ }^{16}$ One potential solution for students' desire for increased guidance is to introduce more PBL- and CBL-style resources that students could utilize or work through without necessarily involving additional group discussion or tutor commitments. Dalhousie's curriculum contains a few excellent examples of attempts to introduce such guidance. During the pediatrics rotation, students are given access to 32 CLIPP cases (Computer-assisted 
Learning in Pediatrics Program), ${ }^{17}$ which ask students to work through common pediatrics presentations, identifying key findings and establishing differential diagnoses, and answering questions throughout. Dermatology uses online cases at the pre-clerkship and clerkship levels both as exercises for students to complete alone, and in preparation for interactive scheduled sessions. Similar strategies have been employed with the teaching of the management of hypertensive disorders of pregnancy at the clerkship level during the obstetrics rotation. If more of these online tools were available and introduced during pre-clerkship years, this could be a great resource for students to use as guidance to optimize their SDL time. Future studies should strive to examine whether the introduction of these curriculum elements leads to improved student uptake and retention of important material, and improvement in their own perceived SDL abilities.

\section{Limitations}

One limitation of this study was the inability to do cross analyses, for example: do the students who report wanting more guidance for SDL also report not using SDL for academic activities? This was a limitation of the survey design and of the Opinio software.

Another limitation was confusion regarding the Likert scale. For example, some students mentioned that they were not sure if responding "most of the time" meant that they spent most of their actual time on a given activity, or if they merely allocated some amount of time to the activity during "most" of their SDL sessions. Future studies could consider assigning percentages of SDL time used for each different type of activity, with a sum total of $100 \%$.

Finally, the response rate of $48.9 \%$, combined with the anonymity of the study also makes it difficult to determine if our respondents are truly representative of the student body as a whole.

\section{Conclusion}

First and second year Dalhousie medical students reported that they used their SDL allocated time for academic activities, and perceived they would benefit from the provision of more specific guidance and tasks to be used during SDL time.

\section{References}

1. Gibbons Maurice. The Self-Directed Learning Handbook: Challenging Adolescent Students to Excel San Francisco; Jossey-Bass, 2002. Print.

2. Spencer JA, Jordan RK. Learner centered approach in medical education. BMJ 1999;318(7193):1280-1283.

3. Frank, JR. (Ed). 2005. The CanMEDS 2005 physician competency framework. Better standards. Better physicians. Better care. Ottawa: The Royal College of Physicians and Surgeons of Canada.
4. Mast TJ, Davis D. Concepts of competence. In: Mast TJ, David D, eds. The Physician as a Learner. Chicago, United States of America: American Medical Association, 1994:139-156.

5. Shin JH, Haynes RB, Johnston ME. Effect of problem-based, self-directed undergraduate education on life long learning. Can Med Assoc J 1993;148(6):969-976.

6. Shokar GS, Shokar NK, Romero CM, Bulik RJ. Self-directed learning: looking at outcomes with medical students. Fam Med 2002;34(3):197-200.

7. Murad MH, Coto-Yglesias F, Varkey P, Prokop LJ, Murad AL. The effectiveness of self-directed learning in health professions education: a systematic review. Medical Education 2010;44(11):1057-1068.

8. Pai KM, Rao KR, Punja D, Kamath A. The effectiveness of self-directed learning (SDL) for teaching physiology to first year medical students. Australas Med J 2014;30(7):448-453.

9. Khabaz Mafinejad M, Aghili R, Emami Z, Malek M, Baradaran H, Taghavinia M, et al. Study guides: effective tools to improve self-directed learning skills of medical students. 2014;52(10):781-785.

10. Al-Shobaili HA, Al-Robaee AA, Al-Zolibani AA, Gabbani SA, Sharaf FK, Inam SN. Utilization of self directed learning allocated times by medical students. Saudi Med J 2010;31(3):333-335.

11. Brydges R, Nair P, Ma I, Shanks D, Hatala R. Directed self-regulated learning vs instructor regulated learning in simulation training. Medical Education 2012;46(7):648-656

12. Guglielmino LM. Development of the Self-Directed Learning Readiness Scale. (Doctoral dissertation, University of Georgia, 1977). Dissertation Abstracts International 1978;38:6467A.

13. Hoban JD, Lawson SR, Mazmanian PE, Best AM, Seibel HR. The SelfDirected Learning Readiness Scale: a factor analysis study. Medical Education 2005;39(4):370-379.

14. Premkumar K, Pahwa P, Banerjee A, Baptiste K, Bhatt H, Lim HJ. Does medical training promote or deter self-directed learning? a longitudinal mixed-methods study. Acad Med 2013;88(11):1754-1764.

15. Abraham RR, Fisher M, Kamath A, Izzati TA, Nabila S, Atikah NN. Exploring first-year undergraduate medical students' self-directed learning readiness to physiology. Adv Phsiol Educ 2011;35(4):393-395.

16. Lee YM, Mann KV, Frank BW. What drives students' self-directed learning in a hybrid PBL curriculum. Adv Health Sci Educ Theory Pract 2010;15(3):425-437.

17. Dell, M. "Computer-assisted Learning in Pediatrics Program". Med-U. < http://www.med-u.org/clipp/> (17 February 2015).

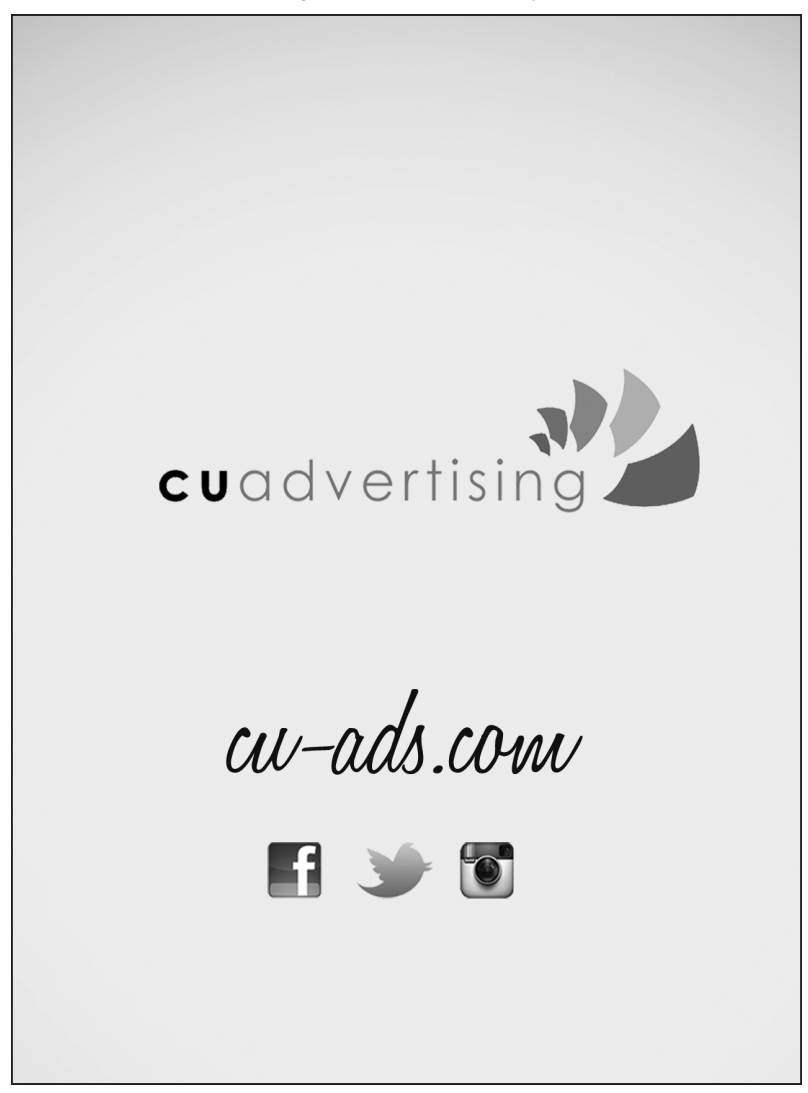

